\title{
Adapted Speed Mechanism for Collision Avoidance in Vehicular Ad hoc Networks Environment
}

\author{
Said Benkirane ${ }^{1}$, Ahmed Jadir ${ }^{2}$ \\ SAEDD Laboratory, School of Technology \\ Cadi Ayyad University \\ Essaouira, Morocco
}

\begin{abstract}
The disrespect of the safety distance between vehicles is the cause of several road accidents. This distance cannot certainly be estimated at random because of some physical rules to be calculated. The more speed gets higher, the more stopping distance increases, mainly in danger case. Thus, the difference between two vehicles must be calculated accordingly. In this paper, we present a mechanism called Adapted Speed Mechanism (ASM) allowing the adaptation of speed to keep the necessary safety distance between vehicles. This mechanism is based on VANET network operation and Multi Agent System integration to ensure communication and collaboration between vehicles. So, it is necessary to perform real-time calculations to make adequate and relevant decisions.
\end{abstract}

Keywords-VANET; multi-agent systems; safety distances; stopping distance; JADE framework

\section{INTRODUCTION}

Keeping enough distance from the vehicle ahead means respecting the balance of traffic. This is the best way to avoid a collision. In many cases, this distance is not respected. For example, on the motorway, where the speed is the fastest, nearly two-thirds of drivers do not always respect the safety distance, which generates several traffic problems.

The use of new communication technologies such as the infrastructure and services offered by the vehicle network (VANET) and the integration of intelligent agents can avoid such problems and improve the quality and safety of driving.

Vehicular Ad hoc Network (VANET) is a particular type of MANET where mobile nodes are smart vehicles equipped with computers (OBU), network cards and sensors [1].

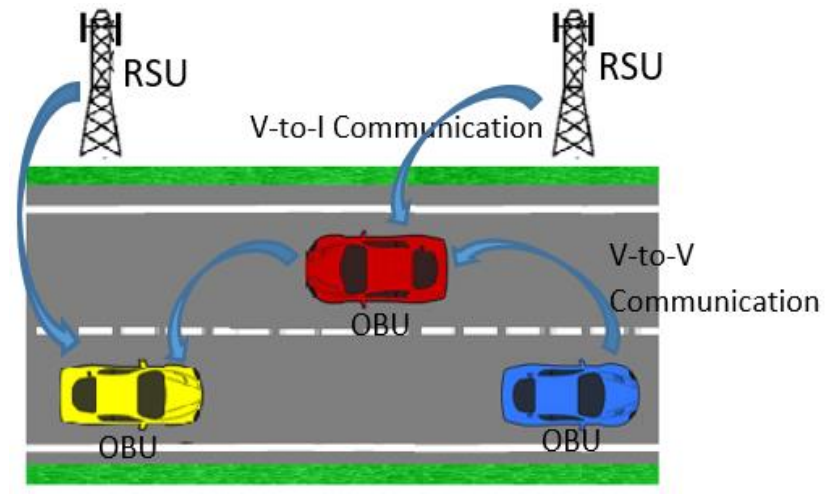

Fig. 1. VANET Network.
Like any other Ad hoc network, vehicles can communicate with each other (for example: exchanging traffic information) or with base stations called roadside unit (RSU) which can be placed all along the roads (seeking information or accessing other networks ...).

VANET networks are based on communication and information exchange between vehicles (V-to-V) [2], and between vehicles and roadside unit (V-to-I) (Examples: signals, intersections lights, etc.) or external network elements (Satellites, WiMAX, LTE...) (Figure 1).

VANETS networks are characterized by great dynamics and mobility nodes, repetitive changes in the network topology and very variable network density. VANETs are expected to implement a variety of wireless technologies such as Dedicated Short Range Communications DSRC, which is a type of Wi-Fi. Other more wireless technologies are Bluetooth, Cellular, Satellite, and WiMAX.

The main applications of VANET networks can be classified in three categories [3][4]:

1) Application in prevention and road safety: VANETs help to prevent collisions and work on the roads, to detect obstacles (fixed or mobile) and to distribute weather information by sending warning messages. It can be used for example to alert a driver to the happening of an accident, and then he can exercise some prudence and forethought when heading to the accident either by changing his direction or doubling his vigilance.

2) Application for traffic optimization and help in driving: Car traffic can be greatly improved through the collection and sharing of data collected by the vehicles, which becomes a technical support for drivers. For example, a car can be notified in case of abnormal slowdown situations [5] (cork, traffic jam, rockslide or works).

3) Applications for driver and passenger comfort: Vehicular networks can also improve the comfort of drivers and passengers. This comfort is illustrated by the internet access, messaging, inter-vehicle chat, etc... [6]. Passengers in the car can play in networks, download MP3 files, send cards to friends and access to other services.

Hence, our ultimate goal is to design a mechanism that could help solve some of the most common road traffic 
problems, such as the safety distance between vehicles, and improving road safety, then making it smarter.

The remainder of this paper is organized as follows: Section 2 gives an overview of Multi-Agent Systems. Section 3 describes our Adapted Speed Mechanism (ASM). Section 4 provides the simulation results. Finally, in Section 5 we conclude our results with a view on the future trends.

\section{Agent And Multi-AgEnt Systems}

An agent is an autonomous physical or abstract entity that is able to act and perceive by itself and about its environment. The agent can communicate with other agents and whose behavior is the result of its observations, its knowledge, and interactions with other agents [7][8]. The agent has its own resources and skills. The agent can both offer services and possibly reproduce some of them.

A multi-agent system (MAS) is a community of autonomous agents evolved in a common environment (Figure 2 ), according to modes of cooperation, competition or even conflict to achieve a global objective [9][10]. These agents constitute a complex system that includes intelligence which could be described as collective.

The agents in a multi-agent system have several important characteristics: [11]

- Autonomy: agents are partially independent and selfaware;

- Local views: no agent has a full global view;

- Decentralization: no agent is designated for controlling.

Multi-agent systems can manifest self-organization, selfdirection and other controlling paradigms. They can also relate complex behaviors even when the individual strategies of all their agents are simple.

MAS tend to find the best solution for their problems without any intervention. There is a high similarity here to physical phenomena, such as energy minimizing, where physical objects tend to reach the lowest energy possible within the physically constrained world [12]. The systems also tend to prevent propagation of faults, self-recover and be fault tolerant, mainly due to the redundancy of components [13].

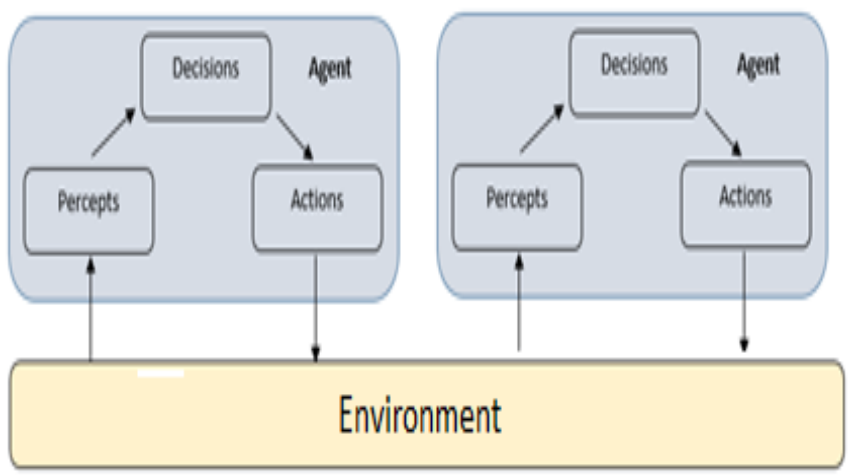

Fig. 2. Multi-Agent System Processes.
MAS are applied in the real world to graphical applications such as computer games. Agent systems have already been used in films. They are used in coordinated defense systems. Other applications include transportation, [14] logistics, [15] graphics and GIS. It is widely advocated for use in networking and mobile technologies, to achieve automatic and dynamic load balancing, high scalability and self-healing networks.

MAS have also applications in the field of artificial intelligence where they reduce the complexity of solving a problem by dividing the necessary knowledge into subsets, by associating an independent intelligent agent with each of these subsets and coordinating the activity of these agents [16]. This is called distributed artificial intelligence.

\section{ADAPTED SPEED MECHANISM (ASM)}

Our mechanism aims to force the driver to drive with adequate speed to keep the safety distance (Figure 3) between two vehicles to avoid a possible collision. This is based on the exchange of information between vehicles via the VANET network infrastructure and by the intelligent agents located in the vehicles that deal with message management and calculation.

Principle: at a time $t$, the vehicle (B) sends its speed, its position, and its length to the vehicle (A) and vice versa ((A) to (B)) and thanks to the calculations carried out based on the kinematics equations, the vehicle (A) will have results concerning the time and distance of a likely collision, and then adjust its speed to avoid it.

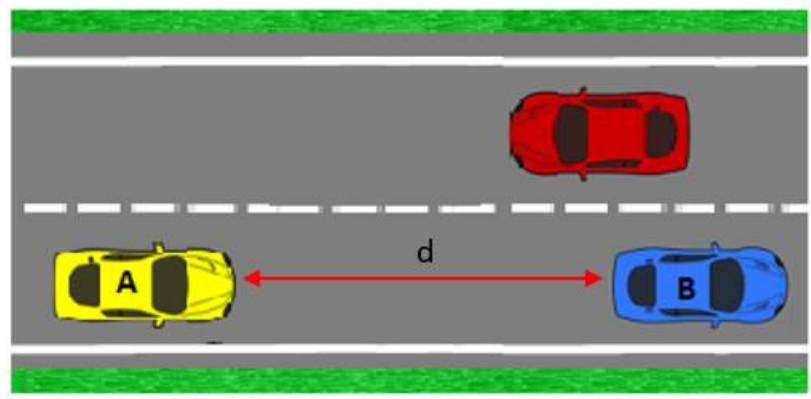

Fig. 3. Safety Distance between Vehicle.

The equations of rectilinear motion uniformly accelerated are given by [17]:

$$
\begin{aligned}
& X_{A}(t)=X_{A}(t=0)+V_{A}(t=o) \times t+\frac{1}{2} a_{A} \times t^{2} \\
& V_{A}(t)=V_{A}(t=o)+a_{A} \times t \\
& V_{A}^{2}(t)=V_{A}^{2}(t=0)+2 \times a_{A} \times \Delta x
\end{aligned}
$$

Where

- $\quad t$ is the elapsed time;

- $X_{A}(t=0)$ is the initial displacement from the origin;

- $X_{A}(t)$ is the displacement from the origin at time $t$;

- $V_{A}(t=o)$ is the initial velocity; 
- $\{\backslash$ displaystyle Imathbf $\{\mathrm{v}\}(\mathrm{t})\} V_{A}(t)$ is the velocity at time $\{$ \displaystyle $\mathrm{t}\} t$

- \{ Idisplaystyle Imathbf $\{\mathrm{a}\}\} a_{A}$ is the uniform rate of acceleration;

- $\Delta x=X_{A}(t)-X_{A}(t=0)$.

A likely collision will occur when:

$X_{A}(t)=X_{B}(t)$

Due to the exchanged positions, the distance separating the two vehicles is given by the following formula:

$d(t)_{A B}=\sqrt{P(t)_{A}^{2}-P(t)_{B}^{2}}$

Suppose Vehicle (A) rolls with speed $V_{A}(t)$, the mechanism consists on calculating the suitable slowing down deceleration for having the appropriate speed at of the safety distance to avoid a possible collision.

The mechanism therefore must make sure to calculate the following equation:

$X_{A}(t)+d(t)_{A B} \leq X_{B}(t)$

Where $d(t)_{A B}$ is the safe distance.

The table below (Table I) shows the recommended safety distances according to the type of road:

TABLE I. SAFETy Distance ACCoRding To SPEED Limit

\begin{tabular}{|l|l|l|}
\hline Road type & Speed limit & Safety distance \\
\hline Urban & $50(\mathrm{~km} / \mathrm{h})$ & $28(\mathrm{~m})$ \\
\hline Extra-Urban & $90(\mathrm{~km} / \mathrm{h})$ & $50(\mathrm{~m})$ \\
\hline Expressway & $110(\mathrm{~km} / \mathrm{h})$ & $62(\mathrm{~m})$ \\
\hline Highway & $130(\mathrm{~km} / \mathrm{h})$ & $73(\mathrm{~m})$ \\
\hline
\end{tabular}

A. Safety Distance

The safety distance is the distance that must be between two vehicles. It depends on the speed at which you ride. But generally, it is admitted that this distance is given by the distance traveled by your car for 2 seconds [18].

In this case:

Safety distance $=V_{A} \times 2$ seconds

\section{B. Stopping Distance}

Stopping distance is the distance traveled by your vehicle between the moment you perceive the danger and the moment when your vehicle is finally stopped. The stopping distance is composed of the reaction path and the braking path [19].

\section{- Reaction path}

The reaction path is the distance your car travels when you see the danger and the moment you press the brakes. A caring and healthy driver has a reaction time of 1 second.

There is a formula to get the reaction path.
Reaction path $=\frac{3 \times V_{A}(\mathrm{~km} / \mathrm{h})}{10}$

- Braking path

The braking path is the distance your vehicle traveled between the moment you pressed the brake and when the car was completely stopped.

We must distinguish two cases. The case of the dry pavement and the case of the wet pavement which considerably lengthens the braking distance.

- Dry pavement:

Braking path $=\frac{V_{A}(\mathrm{~km} / \mathrm{h})}{10} \times \frac{V_{A}(\mathrm{~km} / \mathrm{h})}{10} \times \frac{3}{4}$

- Wet pavement

Braking path $=\frac{V_{A}(\mathrm{~km} / \mathrm{h})}{10} \times \frac{V_{A}(\mathrm{~km} / \mathrm{h})}{10}$

The stopping distance is the addition of the reaction and braking paths. Then:

Stopping distance $=$ Reaction path + Braking path

\section{Simulation AND RESUlts}

To simulate our approach we have developed an application with java language using JADE Framework (Java Agent DEvelopment) [20]. The Topology is a simple road composed of two lanes and consists of vehicles deployed in the different tracks.

As mentioned above, we have created two agents: Vehicle (A) Agent and Vehicle (B) Agent. Each agent is contained in a platform (Container) composed of an Agent Management System (AMS) and a Directory Facilitator (DF) as showed in figure 4. JADE agents use messages that conform to FIPA ACL (FIPA Agent Communication Language) specifications [21].

Thus, the integration of the agents in the vehicles aims at the exchange of messages in real time and made the necessary calculations according to the kinematics equations. Based on agents' properties such as cooperation, coordination and negotiation [22], agents form a distributed and cooperative environment that allows drivers to drive with optimal speeds to avoid possible collisions.

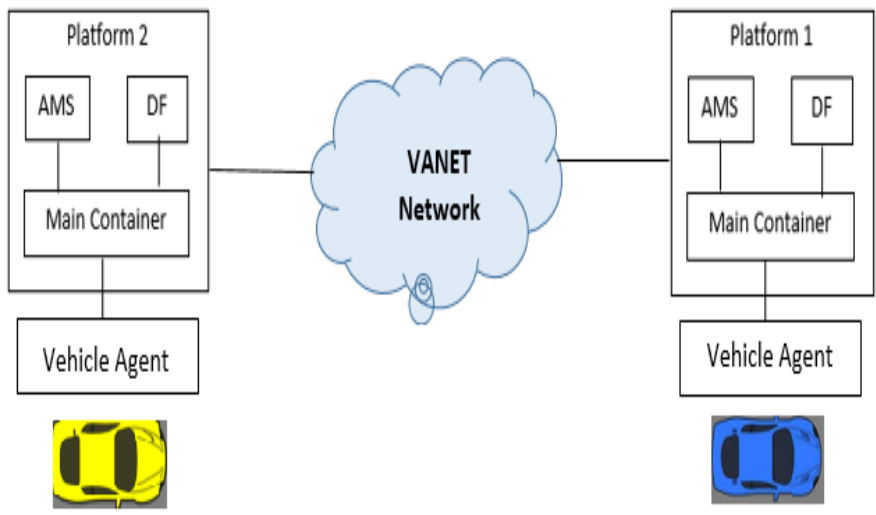

Fig. 4. Architecture of the Proposed Mechanism. 
TABLE II. INITIAL PARAMETERS

\begin{tabular}{|c|l|}
\hline Parameter & Value \\
\hline$V_{A}(t)$ & $30 \mathrm{~m} / \mathrm{s}$ \\
\hline$V_{B}(t)$ & $0 \mathrm{~m} / \mathrm{s}, 10 \mathrm{~m} / \mathrm{s}$ \\
\hline$d(t=0)_{A B}$ & $50(\mathrm{~m})$ \\
\hline
\end{tabular}

We will analyze two situations: the vehicle B is in a stop state, and the second case, the vehicle B rolls with a speed lower than that of vehicle A. We will seek the minimum deceleration necessary to avoid the collision. The table II below shows the initial parameters:

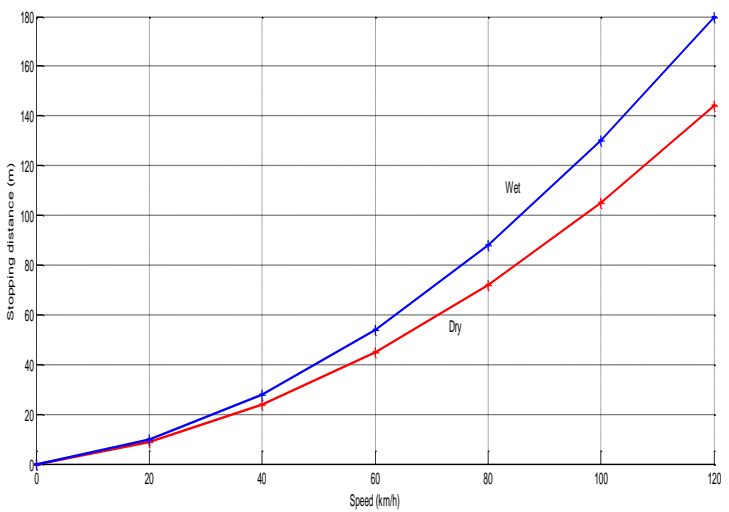

At the moment of collision, the problem is to solve the following system of equations:

$$
\left\{\begin{array}{l}
X_{A}(t=0)+V_{A}(t=o) \times t+\frac{1}{2} a_{A(\min )} \times t^{2}=X_{B}(t) \\
V_{A}(t)=V_{B}(t)
\end{array}\right.
$$

Therefore, we will have the following results:

Figure 5 shows the stopping distances according to the speeds in case of Dry and wet road. We note that as the speed increases the stopping distance increases too. In case of wet road, to stop takes more distance than in case of dry road.

Figure 6 illustrates the different variations of traveled distances related to the function of time and according to different decelerations for the case of fixed vehicle (B) $\left(V_{B}(t)=0\right)$. We notice that for the case of $(\mathrm{a}=0)$ that is to say the vehicle (A) rolls with a constant speed $\left(V_{A}(t)=\right.$ $30 \mathrm{~m} / \mathrm{s})$, the collision between the two vehicles will occur after 1.67 seconds. We also notice that we will never have a collision for a deceleration of $\left(\mathrm{a}=-12 \mathrm{~m} / \mathrm{s}^{2}\right)$ or less. Thus, the recommended deceleration to avoid a possible collision is: $a_{\min }$ $<-9 \mathrm{~m} / \mathrm{s}^{2}$. Based on the results shown in figure 7 above, if the vehicle (B) runs with a speed $\left(V_{B}(t)=0\right)$ and vehicle (A) still runs with a constant speed $\left(V_{A}(t)=30 \mathrm{~m} / \mathrm{s}\right)(\mathrm{a}=0)$, a collision with the vehicle (B) will occur in 2.5 seconds. For cases $\left(\mathrm{a}=-6,-9\right.$, and $\left.-12 \mathrm{~m} / \mathrm{s}^{2}\right)$, a collision will never occur. The recommended optimal deceleration to avoid a possible collision is: $a_{\min }<-4 \mathrm{~m} / \mathrm{s}^{2}$.

Fig. 5. Stopping Distance According to Speed.

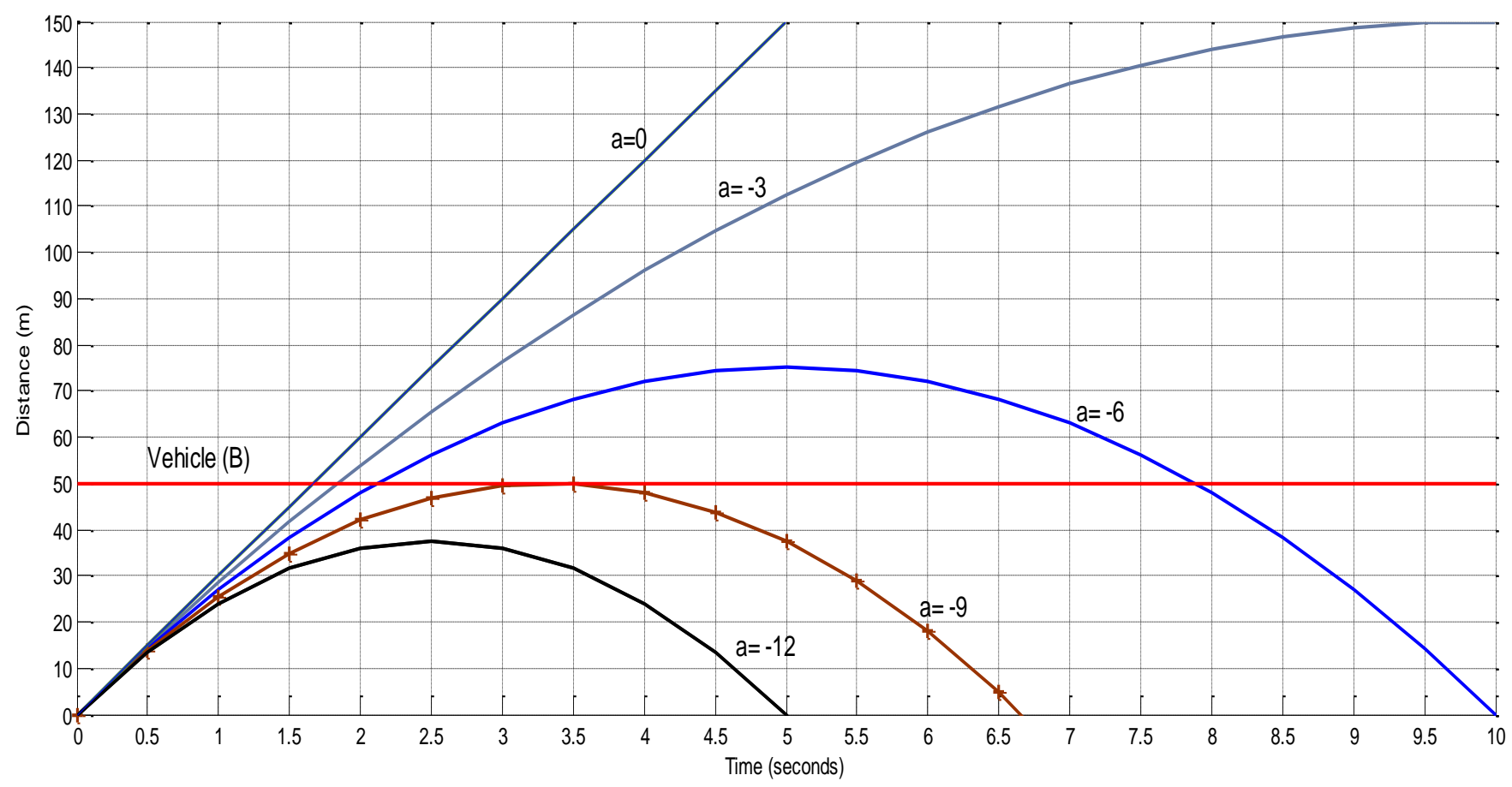

Fig. 6. Stopping Distances According to Different Deceleration and time $\left(V_{B}(t)=0\right)$. 


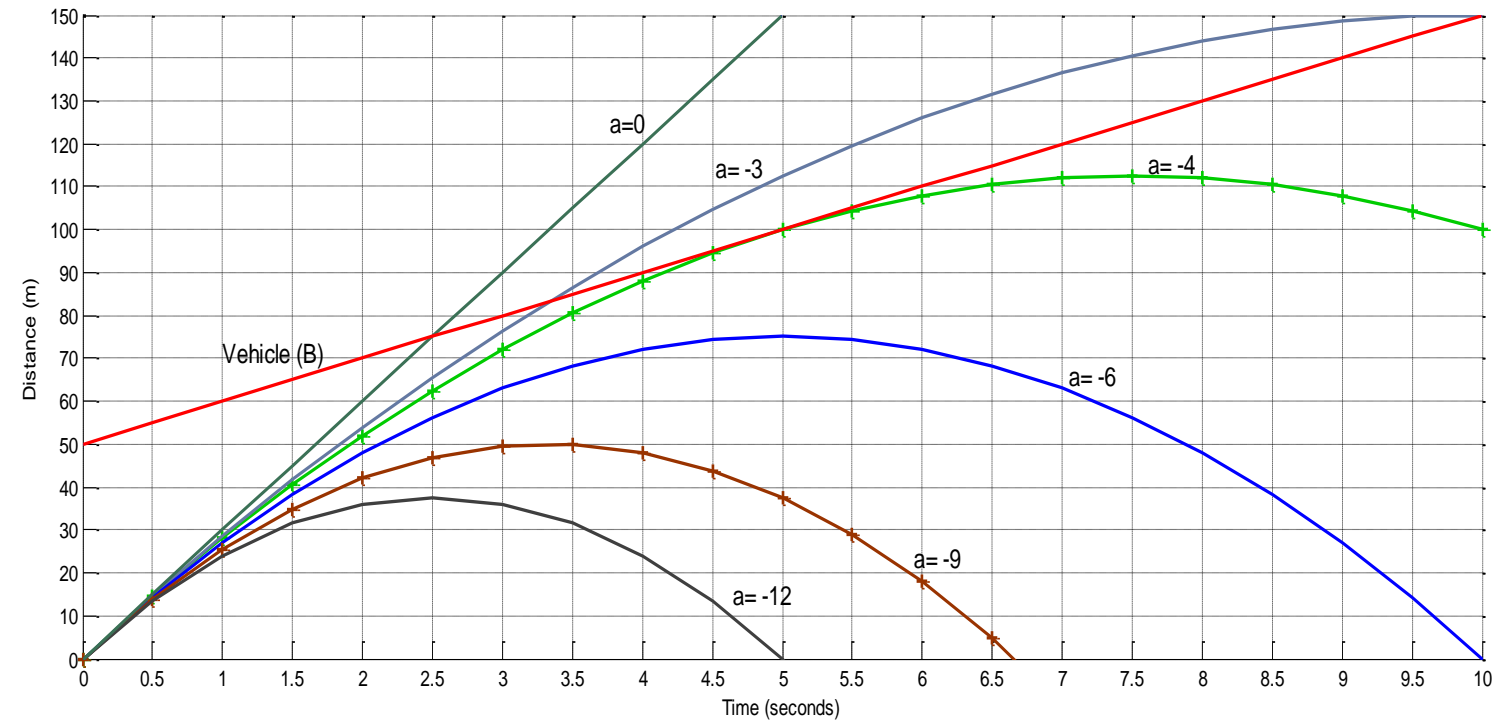

Fig. 7. Stopping Distances According to Different Deceleration and time $\left(V_{B}(t)=10 \mathrm{~m} / \mathrm{s}\right)$.

\section{CONCLUSION}

In this work, we presented a mechanism called Adapted Speed Mechanism (ASM) allowing the adaptation of speed to maintain the recommended safety distance between the vehicles in order to avoid possible accidents. This mechanism is based on VANET network operation and the integration of agents in vehicles. Thus, these agents form an intelligent system for communication and collaboration between vehicles by changing useful and necessary information to ensure driving in good conditions. In future work, we will improve our system to take into account other factors that can influence the calculation of the safety distance such as weather conditions, road conditions as well as vehicles condition.

\section{REFERENCES}

[1] H. Hartenstein and K. Laberteaux, Eds., VANET: Vehicular Applications and Inter-Networking Technologies. Wiley, 2010.

[2] K. C. Dey, A. Rayamajhi, M. Chowdhury, P. Bhavsar, and J. Martin, "Vehicle-to-vehicle (V2V) and vehicle-to-infrastructure (V2I) communication in a heterogeneous wireless network - Performance evaluation," Transportation Research Part C: Emerging Technologies, vol. 68, pp. 168-184, 2016.

[3] Raju Barskar, Meenu Chawla, Vehicular Ad hoc Networks and its Applica-tions in Diversified Fields, International Journal of Computer Applications (0975 8887) Volume 123 No.10, August 2015.

[4] P.Pereira, A. Casaca, J.Rodrigues, V. Soares, J. Triay, C.CervelloPastor," From Delay-Tolerant Networks to Vehicular Delay-Tolerant Networks," Communications Surveys \& Tutorials, IEEE, Vol.14,Issue.4, pp. 1166 -1182,2012.

[5] S.Al-Sultan, M.M. Al-Doori ,A.H. Al-Bayatti , H. Zedan,” A comprehensive survey on vehicular Ad Hoc network," Journal of Network and Computer Applications, Vol. 37, pp.380-392, 2014.

[6] H.Hartenstein, K.P.Laberteaux, "A tutorial survey on vehicular ad hoc networks," Communications Magazine, IEEE, Vol.46, Issue. 6, pp. 164 $171,2008$.

[7] Julie A. Adams. Multiagent Systems: A modern approach to distributed artificial intelligence. AI Magazine, 22(2) :105-108, 2001.

[8] Ferber, J. (1999). Multi-agent systems: An introduction to distributed artificial intelligence. Harlow, UK: Addison-Wesley
[9] Chopra, N., \& Spong, M. W. (2006). Passivity-based control of multiagent systems. In Advances in Robot Control (pp. 107-134). Berlin, Heidelberg: Springer.

[10] Nicholas R.Jennings "On agent-based software engineering" Artificial Intelligence, Volume 117, Issue 2, Pages 277-296, March 2000,

[11] Wooldridge, Michael (2002). An Introduction to MultiAgent Systems. John Wiley \& Sons. p. 366. ISBN 0-471-49691 X

[12] Ferdinand P. Beer, E. Russell Johnston, Jr. Lecture Notes: J. Walt Oler Texas Tech University "VECTOR MECHANICS FOR ENGINEERS: DYNAMICS", Kinematics of Particles. (C) 2007 The McGraw-Hill Companies, Inc.

[13] Orcun. Y, Geylani. K 2014 "A multi-agent system for minimizing energy costs in cement production" Computers in Industry, Volume 65, Issue 7, Pages 1076-1084, September.

[14] Oprea, M.: 2004 “Applications of multi-agent systems, in: Reis, R. (ed.): Information Technology, Kluwer Academic Publishers, Boston Dordrecht London 239-270.

[15] Xiao-Feng Xie, S. Smith, G. Barlow. 2012 "Schedule-driven coordination for real-time traffic network control". International Conference on Automated Planning and Scheduling (ICAPS), São Paulo, Brazil,: 323-331.

[16] Máhr, T. S., Srour, J., De Weerdt, M., Zuidwijk, R. (2010). 2009"Can agents measure up? A comparative study of an agent-based and on-line optimization approach for a drayage problem with uncertainty" Transportation Research Part C: Emerging Technologies. 18: 99. doi:10.1016/j.trc..04.018.

[17] Ponomarev, S., Voronkov, A. (2017) "Multi-agent systems and decentralized artificial superintelligence". arXiv preprint arXiv: 1702.08529.

[18] "BASIC theory of driving The Official Handbook" published by Singapore Traffic Police, on 01.07.2017.

[19] Dr Michael Evans "Maths delivers! Braking distance" Australian Mathematical Sciences Institute (AMSI), 2013.

[20] Bellifemine, F., Poggi, A., \& Rimassa, g. (2001). Developing multiagent systems with a FIPA-compliant agent framework. SoftwarePractice and Experience, 31, 103-128.

[21] FIPA, Geneva, Switzerland, FIPA ACL Message Structure Specification, 2000/08/01 edition (August 2000).

[22] S. Kraus, "Negotiation and cooperation in multi-agent environments," Artificial Intelligence, vol. 94, no. 1-2, pp. 79-98, 1997. 